\title{
Triglyceride glucose index, a marker of insulin resistance, is associated with coronary artery stenosis in asymptomatic subjects with type 2 diabetes
}

Eun Young Lee ${ }^{1}$, Hae Kyung Yang ${ }^{1}$, Joonyub Lee¹, Borami Kang ${ }^{1}$, Yeoree Yang ${ }^{1}$, Seung-Hwan Lee', Seung-Hyun Ko' ${ }^{2}$ Yu-Bae Ahn², Bong Yun Cha', Kun-Ho Yoon ${ }^{1,3}$ and Jae Hyoung Cho ${ }^{1,3^{*}}$

\begin{abstract}
Background: Insulin resistance is one of the most important contributing factors to cardiovascular disease. This study aimed to investigate the association between coronary artery stenosis (CAS) and triglyceride glucose index (TyG index), a simple insulin resistance marker, in asymptomatic subjects with type 2 diabetes.

Methods: We recruited asymptomatic adults with type 2 diabetes but without previous history of coronary heart disease $(n=888)$. Significant CAS was defined as maximum intraluminal stenosis $\geq 70 \%$ by coronary CT angiography. TyG index was calculated as log [fasting triglycerides $(\mathrm{mg} / \mathrm{dl}) \times$ fasting glucose $(\mathrm{mg} / \mathrm{dl}) / 2$ ].

Results: Mean age was $63.8 \pm 9.5$ and $58.9 \%$ of the subjects were men. We analyzed the participants according to the tertile of TyG index. The TyG index was correlated with HOMA-IR $(r=0.397, P<0.001)$, and subjects with higher tertile of TyG index were younger but showed worse clinical and metabolic parameters. The prevalence of CAS was higher in subjects with higher tertile of TyG compared with those with lower tertile of TyG (14\% vs. $7.8 \%, P=0$. 022). On multiple regression analysis, the highest tertile of TyG index was an independent risk factor for CAS after adjustment for other confounders (odds ratio, 3.19 [95 \% Cl, 1.371-7.424]). Subgroup analysis showed that TyG index showed more significant association with CAS in patients with risk factors such as old age, longer duration of diabetes, poor glycemic control, no statin use, and male gender.
\end{abstract}

Conclusion: Higher TyG index is associated with increased risk of CAS in asymptomatic subjects with type 2 diabetes, particularly when they have risk factors for cardiovascular disease.

Trial registration: This study was retrospectively registered in ClinicalTrials. gov with the registration number of NCT02070926 in Feb 23, 2014.

Keyword: Atherosclerosis, Coronary, TyG index, Type 2 diabetes

\section{Background}

Cardiovascular disease (CVD) is a major concern in subjects with type 2 diabetes as it ultimately can lead to severe morbidity and mortality. In type 2 diabetes,

\footnotetext{
*Correspondence: drhoppoer@catholic.ac.kr

'Division of Endocrinology and Metabolism, Department of Internal Medicine, Seoul St. Mary's Hospital, College of Medicine, The Catholic University of Korea, 222, Banpo-daero, Seocho-gu, Seoul 06591, Republic of Korea

${ }^{3}$ Institute of Catholic Ubiquitous Health Care, The Catholic University of Korea, Seoul, Korea

Full list of author information is available at the end of the article
}

macrovascular complications are the primary cause of mortality, accounting for more than $50 \%$ of all death [1]. In addition to the high prevalence of CVD in type 2 diabetes, the recurrence rate of CVD is very high (around $6 \%$ /year) in subjects with type 2 diabetes and prior CVD $[2,3]$. In Korea, the prevalence of ischemic heart disease is about 4 times greater in patients with diabetes compared to those without diabetes [4]. Furthermore, coronary intervention or bypass surgery was performed at frequencies of up to 10-fold higher in patients with type 2 diabetes than those without diabetes 
[4]. Therefore, it is important to identify subjects at high risk for CVD and to manage their condition early, particularly in those with type 2 diabetes.

One of the most important contributing factors to CVD is insulin resistance, which refers to a decreased sensitivity and responsiveness to the metabolic actions of insulin. Insulin resistance predisposes to several disorders such as hyperglycemia, hypertension, and dyslipidemia, all of which are strongly associated with atherosclerosis. In addition, insulin resistance contributes to vasoconstriction, inflammation, and thrombosis, leading to accelerated atherosclerosis. Previous studies have demonstrated an independent association between insulin resistance and clinical or subclinical CVD in both non-diabetic and diabetic subjects [5-8]. In non-diabetic subjects, insulin resistance is associated with the presence of coronary artery diseases and incident CVD [6, 7]. In diabetic subjects, insulin resistance is associated with the thickening of the carotid artery intima-media and incident CVD $[5,8]$.

Recently, a simple and inexpensive approach to evaluate for insulin resistance has been developed [9]. This particular test is based on measuring the product of fasting triglycerides and glucose. Therefore, we aimed to investigate the association between CAS and triglyceride glucose index (TyG index) in asymptomatic subjects with type 2 diabetes. To the best of our knowledge, this is the first study to investigate the association between TyG index and CAD.

\section{Methods}

\section{Study subjects and design}

This study was an observational cohort study based on the CRONOS-ADM Registry (Coronary CT angiography evaluation for clinical outcomes in asymptomatic patients with type 2 diabetes mellitus, registered with ClinicalTrials.gov NCT02070926). We recruited asymptomatic subjects with type 2 diabetes who underwent coronary $\mathrm{CT}$ angiography between Jan 2006 and Dec 2010. In the present study, we included subjects who were aged $\geq 18$ years and had neither a history of ischemic heart disease nor symptoms of angina. We excluded those with type 1 diabetes, cardiac transplantation, ventricular or supraventricular arrhythmias, and ischemic heart disease such as coronary artery disease, myocardial infarction, and coronary revascularization. Subjects who had elevated triglyceride levels $(>500 \mathrm{mg} / \mathrm{dl})$ or had taken antianginal medication or medications lowering primarily triglycerides (fenofibrate, omega-3) were also excluded. We measured blood glucose, fasting insulin, and lipid profiles. HOMA-IR was calculated as insulin $(\mathrm{mU} / \mathrm{l}) \times$ (glucose $[\mathrm{mg} / \mathrm{dl}] \times 0.055) / 22.5$ [10]. The TyG index was calculated by the logarithm of fasting triglyceride $\mathrm{x}$ fasting glucose/2 [9]. Metabolic syndrome was assessed in those whose waist circumference data were available $(n=280)$. Metabolic syndrome was defined according to the revised criteria of the National Cholesterol Education Program Adult Treatment panel III (NCEP-ATP III) [11]. This study protocol was conducted in full compliance with the Declaration of Helsinki and was approved by the institutional review board of Seoul St. Mary's Hospital, The Catholic University of Korea (No. KC15RISI0955). The written informed consent from the participants was waived by the institutional review board as only deidentified data were accessed and analyzed.

\section{Imaging protocol and analysis}

Coronary CT angiography imaging was obtained using a 64-slice, dual source CT scanner (SOMATOM Definition, Siemens, Forchheim, Germany). All patients were in normal sinus rhythm and if the heart rate was faster than 70 beats per minute (bpm), intravenous beta blocker (esmolol) was administered at a dose of $3 \mathrm{mg}$ at 5-min intervals up to three times. Images were obtained before and after administration of $80-110 \mathrm{~mL}$ of iodinated contrast (Lomeron 350, iomeprol, Bracco, Milano, Italy). All scans were performed using ECG-controlled tube current modulation and the estimated radiation dose was less than $14 \mathrm{mSv}$. To obtain coronary artery images, all images were reconstructed immediately after scan completion.

Coronary CT angiography images were analyzed by two experienced radiologists. According to the guidelines of the Society of Cardiovascular Computed Tomography [12], each segment of the coronary artery was scored visually for the presence of a coronary plaque using a 16-segment coronary artery model in an intentto-diagnose fashion. All analysis was performed only on segments with a diameter $\geq 1.5 \mathrm{~mm}$. Coronary plaques were defined as structures $\geq 1 \mathrm{~mm}^{2}$ within or adjacent to the coronary artery lumen, which were characterized from the vessel lumen or surrounding pericardial tissues. Significant CAS was diagnosed when there was severe coronary artery stenosis, or when the maximum intraluminal stenosis in any of the segments of the major epicardial coronary arteries was greater than $70 \%$ [13].

\section{Statistical analysis}

Data are presented as mean \pm standard deviations and number (percentages). ANOVA and post hoc analysis were performed to compare groups according to tertiles of TyG index. For nonparametric variables, data are presented as median (interquartile range) and the KruskalWallis test was performed to compare three groups. Categorical variables were analyzed using the $\chi^{2}$ or MantelHaenszel $\chi^{2}$ test. In order to adjust for age and sex, partial Pearson's correlation coefficient (R) was used to assess the relationships between the two variables. Multivariate logistic regression analysis was performed in order to control for confounding factors. Subgroup analysis was performed after categorizing the subjects 
according to age, sex, duration of diabetes, glycemic control, hypertension, and statin use. In subgroup analysis, odds ratio was compared between the highest and lowest tertile of TyG index. All statistical analyses were performed using SPSS (version 20.0; IBM SPSS, NY, USA). A $P$ value $<0.05$ was considered statistical significant.

\section{Results}

\section{Baseline characteristics according to the tertiles of TyG index}

Mean age was $63.9 \pm 9.5$ years and $58.9 \%(n=523)$ of the study population $(n=888)$ were male. We analyzed participants according to tertiles of TyG index. Subjects in the higher tertile of TyG index were younger but had greater BMI, WC, WHR, and blood pressure (Table 1).
Glycemic indices, such as plasma glucose and glycated hemobgloin, and serum uric acid levels were higher amongst subjects with higher tertile of TyG index. In addition, subjects with higher tertile of TyG index showed higher total and LDL-cholesterol levels but lower HDL-cholesterol levels. There were no differences in the duration of diabetes and renal function according to the tertiles of TyG index. In subjects with higher tertile of TyG index, more proportion used insulin and metformin while $\alpha$-glucosidase inhibitor and antiplatelet agent use were less common. There was no difference in statin use according to the tertiles of TyG index. At baseline electrocardiography (ECG), 62 participants showed left ventricular hypertrophy $(n=62,7.0 \%)$. Other frequently observed changes in ECG were as

Table 1 Baseline characteristics of study population according to the tertiles of TyG index

\begin{tabular}{|c|c|c|c|c|}
\hline & Lowest tertile $(n=293)$ & Mid tertile $(n=296)$ & Highest tertile $(n=299)$ & $P$ value \\
\hline Age (year) & $65.4 \pm 8.6$ & $64.2 \pm 9.3$ & $62.0 \pm 10.2^{*} \dagger$ & $<0.001$ \\
\hline Male (\%) & $180(61.4)$ & $172(58.1)$ & $171(57.2)$ & 0.295 \\
\hline Duration of diabetes (year) & $12.3 \pm 9.3$ & $12.2 \pm 9.6$ & $11.8 \pm 9.3$ & 0.779 \\
\hline BMI $\left(\mathrm{kg} / \mathrm{m}^{2}\right)$ & $23.2 \pm 3.1$ & $24.6 \pm 3.0^{*}$ & $24.9 \pm 3.3^{*}$ & $<0.001$ \\
\hline$W C(\mathrm{~cm})$ & $85.6 \pm 8.5$ & $89.8 \pm 11.3^{*}$ & $89.8 \pm 8.5^{*}$ & 0.005 \\
\hline WHR $(\mathrm{cm})$ & $0.9 \pm 0.1$ & $0.9 \pm 0.1$ & $1.0 \pm 0.1^{*}$ & 0.010 \\
\hline $\mathrm{SBP}(\mathrm{mmHg})$ & $123.7 \pm 14.5$ & $126.6 \pm 13.3$ & $127.0 \pm 15.8^{*}$ & 0.016 \\
\hline $\mathrm{DBP}(\mathrm{mmHg})$ & $73.2 \pm 9.7$ & $74.8 \pm 9.5$ & $77.0 \pm 9.7^{*}+$ & $<0.001$ \\
\hline TyG index & $8.2 \pm 0.3$ & $8.9 \pm 0.2^{*}$ & $9.7 \pm 0.4^{*}+$ & $<0.001$ \\
\hline HOMA-IR & $2.1(1.3-3.7)$ & $3.9(2.1-7.1) *$ & $5.2(3.2-10.1) *+$ & $<0.001$ \\
\hline $\mathrm{FPG}(\mathrm{mg} / \mathrm{dL})$ & $117.1 \pm 28.8$ & $138.8 \pm 31.6^{*}$ & $188.4 \pm 71.1^{*} \dagger$ & $<0.001$ \\
\hline PPG (mg/dL) & $185.6 \pm 65.8$ & $205.6 \pm 76.0^{*}$ & $245.6 \pm 83.6^{*}+$ & $<0.001$ \\
\hline HbA1c (\%) & $7.4 \pm 1.8$ & $7.7 \pm 1.9$ & $8.8 \pm 2.0^{*}+$ & $<0.001$ \\
\hline Total cholesterol (mg/dL) & $155.5 \pm 30.7$ & $165.9 \pm 32.8^{*}$ & $182.9 \pm 42.5^{*}+$ & $<0.001$ \\
\hline Triglyceride (mg/dL) & $68(54-83)$ & $115(94-138)^{*}$ & $188(145-253) *+$ & $<0.001$ \\
\hline HDL cholesterol (mg/dL) & $52.0 \pm 13.4$ & $46.5 \pm 10.2^{*}$ & $44.2 \pm 10.4^{*}$ & $<0.001$ \\
\hline LDL cholesterol (mg/dL) & $89.9 \pm 27.4$ & $96.1 \pm 29.7$ & $97.4 \pm 37.7^{*}$ & 0.009 \\
\hline Estimated GFR (mL/min/1.73 $\left.\mathrm{m}^{2}\right)$ & $95.7 \pm 24.6$ & $93.8 \pm 25.1$ & $93.1 \pm 24.2$ & 0.125 \\
\hline Uric acid (mg/dL) & $4.9 \pm 1.3$ & $5.0 \pm 1.4$ & $5.2 \pm 1.6$ & 0.046 \\
\hline Insulin, n (\%) & $59(20.1)$ & $66(22.3)$ & $83(27.8)$ & 0.028 \\
\hline Sulfonylurea, n (\%) & $117(39.9)$ & $127(42.9)$ & $109(36.5)$ & 0.383 \\
\hline Meglitinide, n (\%) & $39(13.3)$ & $33(11.1)$ & $35(11.7)$ & 0.551 \\
\hline Metformin, n (\%) & $161(54.9)$ & $193(65.3)$ & $202(67.6)$ & 0.002 \\
\hline Thiazolidinedione, n (\%) & $8(2.7)$ & $8(2.7)$ & $12(4.0)$ & 0.370 \\
\hline a-glucosidase inhibitor, n (\%) & $69(23.5)$ & $63(21.3)$ & $36(12.0)$ & $<0.001$ \\
\hline DPP-IV inhibitor, n (\%) & $19(6.5)$ & $25(8.4)$ & $22(7.4)$ & 0.689 \\
\hline Antiplatelet agent, n (\%) & $192(66.2)$ & $173(58.6)$ & $153(51.7)$ & $<0.001$ \\
\hline Statin, n (\%) & $151(51.5)$ & $176(59.5)$ & $138(53.8)$ & 0.580 \\
\hline
\end{tabular}

Data are expressed as mean $\pm \mathrm{SD}$, median (interquartile range), or $\mathrm{N}$ (\%). ACR, albumin to creatinine ratio; BMI body mass index, $D B P$ diastolic blood pressure, $D P P$ IV dipeptidyl peptidase IV, FPG fasting plasma glucose, GFR glomerular filtration rate, HDL high density lipoprotein, HOMA-IR homeostasis model assessment insulin resistance, $L D L$ low density lipoprotein, $P P G$ postprandial plasma glucose, SBP systolic blood pressure, WC waist circumference, $W H R$ waist to hip ratio. * vs. lowest tertile, + vs. mid tertile 
follows: first degree atrioventricular block $(n=37,4.2 \%)$, right bundle branch block $(n=29,3.3 \%)$, atrial or ventricular premature complex $(n=22,2.5)$, atrial fibrillation $(n=3,0.3 \%)$, T wave inversion $(n=3,0.3 \%)$, ST depression $(n=2,0.2 \%)$, and Q wave $(n=1,0.1 \%)$. The frequencies of these ECG changes were not different across the tertile of TyG index.

\section{Association of TyG index with HOMA-IR and metabolic syndrome}

HOMA-IR values increased significantly according to the tertiles of TyG index (Table 1). After adjusting for age and sex, the TyG index was significantly correlated with the HOMA-index (Fig. 1). The prevalence of metabolic syndrome was significantly higher in subjects with the highest tertile of TyG index compared to subjects of the lowest tertile $(84.1 \%$ vs $39.4 \%, P<0.001)$. In addition, features of metabolic syndrome, such as hypertension, abdominal obesity, or low HDL-cholesterol were more common amongst subjects with the higher tertiles of TyG index (all $P<0.05$ ).

\section{Association of TyG index with CAS}

Of the 888 subjects with asymptomatic type 2 diabetes, 109 (12.3\%) were found to have significant CAS on MDCT. The prevalence of significant CAS was higher in subjects with the higher tertile of TyG index (14\% for the highest tertile of TyG index vs $7.8 \%$ for the lowest tertile of TyG index, $P=0.022$ ). On multiple regression analysis (Table 2), the higher tertile of TyG index was significantly associated with CAS after adjusting for age and sex (odds ratio, 2.36 [95 \% GI, 1.370-4.078] for mid tertile and 2.50 [1.432-4.348] for the highest tertile of

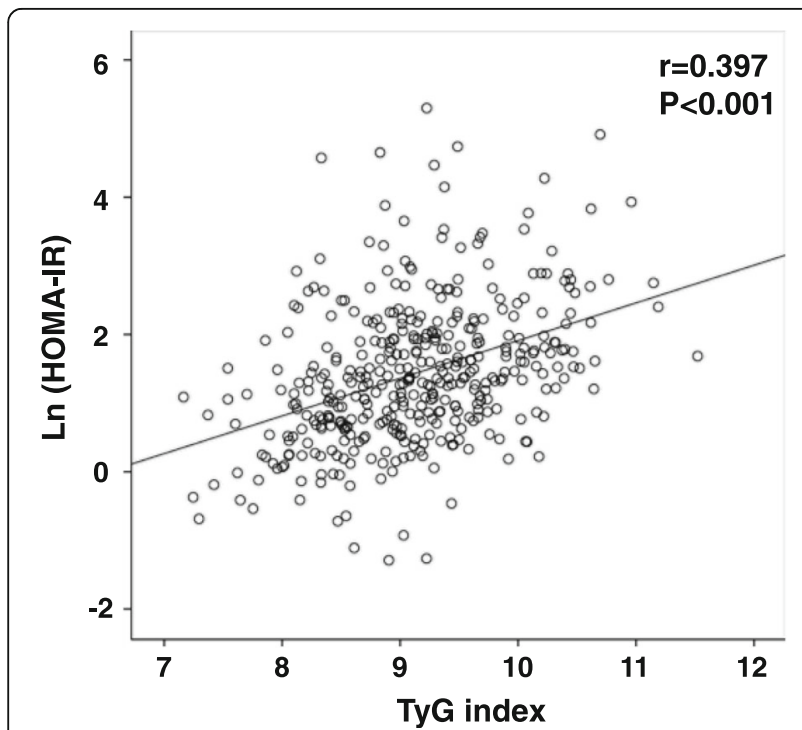

Fig. 1 Correlation between TyG index and HOMA-IR after adjustment for age and sex
TyG index). After further adjustment for other confounding factors, such as glycemic control, blood pressure, LDL-cholesterol and medications, the highest tertile of TyG index was an independent risk factor for CAS (odds ratio, 3.19 [95 \% CI, 1.371-7.424]). As shown in Fig. 2, subgroup analysis showed that subjects aged $\geq 60$ years and with the highest tertile of TyG index had an independent association with CAS (odds ratio, 2.78 [95 \% CI, 1.085-7.124]). In addition, subjects with male gender, longer duration of diabetes, and poor glycemic control had higher odds ratios than those without these risk factors. Subjects receiving insulin also showed higher odds ratio compared to those without insulin therapy. Meanwhile, subjects receiving antiplatelet agent or statin had lower odds ratios compared to those without these medications.

\section{Discussion}

In the present study, we observed that the TyG index was correlated with HOMA-IR and that there were significant differences in cardiometabolic parameters, including the presence of metabolic syndrome, according to the TyG index. In addition, we demonstrated that the higher TyG index is associated with an increased risk of CAS in asymptomatic subjects with type 2 diabetes. Furthermore, subgroup analysis showed that the TyG index is significantly associated with CAS in subjects with cardiovascular risk factors such as older age, longer duration of diabetes, poor glycemic control, hypertension, absence of statin or antiplatelet agents use, and male gender. This suggests that the TyG index may be used as a marker for insulin resistance and, importantly, to help identify subjects at high risk of CVD.

Insulin resistance is a hallmark of type 2 diabetes and even precedes the disease for several years [14]. Many studies indicate that insulin resistance per se and associated disorders contribute to the development of CVD in nondiabetic as well as diabetic subjects. It is well known that insulin resistance is related to cardiometabolic risk factors, such as hyperglycemia, dyslipidemia, and hypertension. In addition, inflammatory and pro-coagulant properties of insulin resistance may also lead to accelerated atherosclerosis [14, 15]. For these reasons, insulin resistance has been considered as not only a pathogenic cause but also a predictor of CVD in type 2 diabetes. Several prospective studies have shown that insulin resistance predicts incident CVD in both the general and diabetic subjects $[5,7,16,17]$. In the general population, insulin resistance, presented as HOMA-IR or metabolic syndrome, was an independent predictor of CVD after adjusting for other risk factors [7, 16, 17]. Similar results were observed in subjects with type 2 diabetes and a hazard ratio for CVD was significantly greater in 
Table 2 Multiple regression analysis for coronary artery stenosis

\begin{tabular}{|c|c|c|c|c|c|c|c|c|}
\hline & Model 1 & & Model 2 & & Model 3 & & Model 4 & \\
\hline TyG index tertile & OR $(95 \% \mathrm{Cl})$ & $P$ value & OR $(95 \% \mathrm{Cl})$ & $P$ value & OR $(95 \% \mathrm{Cl})$ & $P$ value & OR (95 \% Cl) & $P$ value \\
\hline Lowest & 1 (reference) & & 1 (reference) & & 1 (reference) & & 1 (reference) & \\
\hline Mid & $2.36(1.370-4.078)$ & 0.002 & $2.28(1.313-3.967)$ & 0.003 & $1.71(0.729-3.991)$ & 0.219 & $1.63(0.674-3.924)$ & 0.279 \\
\hline Highest & $2.50(1.432-4.348)$ & 0.001 & $2.54(1.418-4.544)$ & 0.002 & $3.42(1.519-7.709)$ & 0.003 & $3.19(1.371-7.424)$ & 0.007 \\
\hline$P$ for trend & 0.002 & & 0.002 & & 0.002 & & 0.006 & \\
\hline
\end{tabular}

Model 1: adjusted for age and sex

Model 2: model + adjusted for $\mathrm{HbA1c}$ and duration of diabetes

Model 3: model 2 + adjusted for systolic blood pressure, LDL cholesterol, estimated GFR, uric acid and smoking

Model 4: model 3 + adjusted for insulin, oral hypoglycemic agents, antiplatelet agents, antihypertensive medication, and statin

$\mathrm{Cl}$ confidence interval, GFR glomerular filtration rate, OR odds ratio

subjects with diabetes compared with those without diabetes $[5,17]$.

In accordance with previous studies, we observed that the TyG index, a marker for insulin resistance, was significantly associated with CAS in asymptomatic subjects with type 2 diabetes. The TyG index was first introduced as a surrogate marker for insulin resistance by GuerreoRomero [9]. The TyG index has shown direct correlation with insulin resistance, as assessed by hyperinsulinemic euglycemic clamp or insulin-mediated glucose uptake $[18,19]$. In a Brazilian population, TyG index showed better performance for assessing insulin resistance than the HOMA-IR [20]. The TyG index showed similar correlation with insulin resistance regardless of sex, obesity, and diabetes [18]. This suggests that the TyG index might be used in various populations as a biomarker of insulin resistance. In addition, previous studies have revealed that the TyG index has an independent association with incident diabetes and carotid atherosclerosis [20-22]. In the present study, we observed that the TyG index was correlated with HOMA-IR and associated with an increased prevalence of metabolic syndrome. Taken together, the TyG index might be useful for the identification of subjects with insulin resistance and associated disorders.

Several potential mechanisms have been suggested to explain the correlation between TyG index and insulin resistance. Increased triglyceride levels can lead to elevated free fatty acids and, thus, increased flux of free fatty acids from adipose to nonadipose tissue, which

\begin{tabular}{|c|c|c|c|}
\hline Characteristics & & Odds Ratio (95\% Cl) & $P$ value \\
\hline Age $\geq 60$ years & 1 & $2.78(1.085-7.124)$ & 0.033 \\
\hline Age $<60$ years & 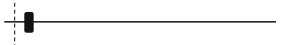 & $2.16(0.227-20.594)$ & 0.502 \\
\hline Male & & $3.72(1.294-10.701)$ & 0.015 \\
\hline Female & 1 & 3.35 (0.697-16.079) & 0.131 \\
\hline Duration $>20$ years & & $5.15(0.985-26.912)$ & 0.052 \\
\hline Duration $\leq 20$ years & 1 & $2.98(0.948-9.364)$ & 0.062 \\
\hline $\mathrm{HbA} 1 \mathrm{c} \geq 7 \%$ & & $5.48(1.619-18.556)$ & 0.006 \\
\hline HbA1c $<7 \%$ & 1 & $1.68(0.257-10.961)$ & 0.589 \\
\hline HTN (+) & 1 & $3.02(0.982-9.254)$ & 0.054 \\
\hline HTN (-) & & $7.14(1.461-34.901)$ & 0.015 \\
\hline Insulin use (+) & -1 & $10.18(1.502-69.002)$ & 0.017 \\
\hline Insulin use (-) & & $2.44(0.865-6.855)$ & 0.092 \\
\hline Antiplatelet agent ( + ) & -1 & $2.71(0.976-7.498)$ & 0.056 \\
\hline Antiplatelet agent (-) & & $5.28(1.048-26.632)$ & 0.044 \\
\hline Statin use $(+)$ & -1 & $2.60(0.846-7.988)$ & 0.095 \\
\hline Statin use (-) & 1 & $4.10(1.013-16.587)$ & 0.048 \\
\hline
\end{tabular}

Fig. 2 Subgroup analysis for coronary artery stenosis. Odds ratio of the highest tertile of TyG index was presented in comparison with the odds ratio of the lowest tertile of TyG index 
may induce insulin resistance [23, 24]. Previous studies have demonstrated that higher levels of triglycerides in the liver and muscle may interfere with glucose metabolism in each target organ [25-27]. These findings support the importance of triglycerides in the pathogenesis of insulin resistance and its possibility to be used as a surrogate marker for insulin resistance. Recently, there have been growing evidence that some nutraceuticals in common diet lower plasma lipid levels and, thus, reduce the overall cardiovascular risk [28]. In terms of TyG index, it might be postulated that the effects of these nutraceuticals on cardiovascular risk would be mediated by improving insulin resistance.

Of note, the association between TyG index and CAS was more significant in subjects with cardiovascular risk factors such as older age, longer duration of diabetes, poor glycemic control, lack of antiplatelet agent or statin use, and male gender. This implies that greater insulin resistance, as represented by the TyG index, makes subjects more vulnerable to risk factors related to CVD. Consistent with our results, high triglyceride levels has been reported to be a predictor of incident or recurrent CVD in subjects with type 2 diabetes [2, 29]. To prevent CVD, aggressive management of modifiable risk factors such as hyperglycemia, hypertension, or dyslipidemia in subjects with cardiovascular risk factors and high TyG index may be necessary. In contrast to other risk factors, the association between TyG index and CAS was less significant in subjects with hypertension. In our study, more than $50 \%$ of subjects with hypertension had taken angiotensin receptor blocker or beta blocker. This may contribute to the different results in subjects with hypertension.

This study has several limitations. Firstly, as this study has cross-sectional observation study design, we could not evaluate for a direct causal relationship. Secondly, we compared the TyG index with HOMA-IR rather than the hyperinsulinemic euglycemic clamp, a gold standard method for measuring insulin resistance. However, previous studies have shown similar associations between the TyG index and insulin resistance, as measured by the hyperinsulinemic euglycemic clamp, in a wide range of subjects $[18,20]$. Thirdly, the TyG index may not be used in subjects with extremely high triglyceride levels or in those taking medications that lower mainly triglycerides. As more than $50 \%$ of the participants had taken statins, we only excluded subjects taking fenofibrate or omega-3. Lastly, we could not adjust for alcohol, fruits, juices, and other simple sugars consumption, which can affect blood triglyceride levels. However, the measurement of CAS by coronary CT angiography, as opposed to surrogate markers such as intima-media thickness, is a strength of this study. In addition, we analyzed data with a relatively large sample size of asymptomatic subjects with type 2 diabetes. One of the advantages of the TyG index is that both the triglyceride and glucose are usually measured in routine clinical practice and is, therefore, readily available. The TyG index can also be obtained by a simple equation and is less costly than the insulin measurement.

\section{Conclusions}

In conclusion, the TyG index is associated with an increased risk of CAS in asymptomatic subjects with type 2 diabetes, particularly when they have other risk factors. The TyG index may be used as a marker for insulin resistance and help identify subjects at high risk of CVD in asymptomatic subjects with type 2 diabetes. Further prospective studies are needed to investigate whether TyG index can predict cardiovascular events in these subjects.

\section{Abbreviations \\ ACR: Albumin to creatinine ratio; ANOVA: Analysis of variance; BMI: Body mass index; CAS: Coronary artery stenosis; Cl: Confidence interval; CRONOS- ADM: Coronary CT angiography evaluation for clinical outcome in asymptomatic patients with type 2 diabetes; CT: Computed tomography; CVD: Cardiovascular disease; DBP: Diastolic blood pressure; DPP-IV: Dipeptidyl peptidase IV; ECG: Electrocardiography; FPG: Fasting plasma glucose; GFR: Glomerular filtration rate; HDL: High density lipoprotein; HOMA- IR: Homeostasis model assessment of insulin resistance; LDL: Low density lipoprotein; NCEP-ATP III: National cholesterol education program adult treatment panel III; OR: odds ratio; PPG: Postprandial plasma glucose; SBP: Systolic blood pressure; TyG index: Triglyceride-to-glucose index; WC: Waist circumference;}

\section{Acknowledgements}

Not applicable.

\section{Funding}

There is no funder for this study.

\section{Availability of data and material}

Data will not be shared with a reason that the institutional review board of Seoul St. Mary's Hospital, The Catholic University of Korea approved this study in case of limited access to data only for designated researchers.

\section{Authors' contributions}

EYL analyzed and interpreted the data and wrote the manuscript. HKY, BK, and YY performed clinical data collection and analysis. SHL, SHK, YBA, BYC, and $\mathrm{KHY}$ assisted with data analysis and interpretation and revised the manuscript. JHC designed the study, analyzed and interpreted data, and revised the manuscript. All authors read and approved the final manuscript.

\section{Competing interests}

The authors declare that they have no competing interests.

\section{Consent for publication \\ Not applicable.}

\section{Author details}

${ }^{1}$ Division of Endocrinology and Metabolism, Department of Internal Medicine, Seoul St. Mary's Hospital, College of Medicine, The Catholic University of Korea, 222, Banpo-daero, Seocho-gu, Seoul 06591, Republic of Korea. ${ }^{2}$ Division of Endocrinology and Metabolism, Department of Internal Medicine, St. Vincent's Hospital, College of Medicine, The Catholic University of Korea, Seoul, Korea. Institute of Catholic Ubiquitous Health Care, The Catholic University of Korea, Seoul, Korea. 
Received: 4 May 2016 Accepted: 2 September 2016 Published online: 15 September 2016

\section{References}

1. Morrish N, Wang S-L, Stevens L, Fuller J, Keen H, Group WMS. Mortality and causes of death in the WHO Multinational Study of Vascular Disease in Diabetes. Diabetologia. 2001:44(2):S14-21.

2. Giorda CB, Avogaro A, Maggini M, Lombardo F, Mannucci E, Turco S, Alegiani SS, Raschetti R, Velussi M, Ferrannini E. Recurrence of Cardiovascular Events in Patients With Type 2 Diabetes Epidemiology and risk factors. Diabetes Care. 2008;31(11):2154-9.

3. Nathan DM, Meigs J, Singer DE. The epidemiology of cardiovascular disease in type 2 diabetes mellitus: how sweet it is... or is it? Lancet. 1997:350:54-9.

4. Association KD. Korean diabetes fact sheet 2015 [Internet]. Seoul: Korean Diabetes Association; 2015. cited 2015 Dec 8.

5. Bonora E, Formentini G, Calcaterra F, Lombardi S, Marini F, Zenari L, Saggiani F, Poli M, Perbellini S, Raffaelli A. HOMA-estimated insulin resistance is an independent predictor of cardiovascular disease in type 2 diabetic subjects prospective data from the Verona Diabetes Complications Study. Diabetes Care. 2002;25(7):1135-41.

6. Bressler P, Bailey S, Matsuda M, DeFronzo R. Insulin resistance and coronary artery disease. Diabetologia. 1996;39(11):1345-50.

7. Hanley AJ, Williams K, Stern MP, Haffner SM. Homeostasis model assessment of insulin resistance in relation to the incidence of cardiovascular disease the San Antonio heart study. Diabetes Care. 2002;25(7):1177-84.

8. Bonora E, Tessari R, Micciolo R, Zenere M, Targher G, Padovani R, Falezza G, Muggeo M. Intimal-medial thickness of the carotid artery in nondiabetic and NIDDM patients: relationship with insulin resistance. Diabetes Care. 1997;20(4):627-31.

9. Simental-Mendía LE, Rodríguez-Morán M, Guerrero-Romero F. The product of fasting glucose and triglycerides as surrogate for identifying insulin resistance in apparently healthy subjects. Metab Syndr Relat Disord. 2008; 6(4):299-304.

10. Matthews D, Hosker J, Rudenski A, Naylor B, Treacher D, Turner R. Homeostasis model assessment: insulin resistance and $\beta$-cell function from fasting plasma glucose and insulin concentrations in man. Diabetologia. 1985:28(7):412-9.

11. Grundy S, Cleeman J, Daniels S, Donato K, Eckel R, Franklin B, Gordon D, Krauss R, Savage P, Smith S. American Heart Association; National Heart, Lung, and Blood Institute. Diagnosis and management of the metabolic syndrome: an American Heart Association/National Heart, Lung, and Blood Institute Scientific Statement. Circulation. 2005;112(17):2735-52.

12. Raff GL, Abidov A, Achenbach S, Berman DS, Boxt LM, Budoff MJ, Cheng V, DeFrance T, Hellinger JC, Karlsberg RP. SCCT guidelines for the interpretation and reporting of coronary computed tomographic angiography. J Cardiovasc Comput Tomogr. 2009;3(2):122-36.

13. Arbab-Zadeh A, Hoe J. Quantification of coronary arterial stenoses by multidetector CT angiography in comparison with conventional angiography: methods, caveats, and implications. J Am Coll Cardiol Img. 2011:4(2):191-202.

14. DeFronzo RA. From the triumvirate to the ominous octet: a new paradigm for the treatment of type 2 diabetes mellitus. Diabetes. 2009;58(4):773-95.

15. Mills JD, Grant PJ. Insulin resistance, haemostatic factors and cardiovascular risk. Br J Diab Vasc Dis. 2002;2(1):19-26.

16. Jeppesen J, Hansen TW, Rasmussen S, Ibsen H, Torp-Pedersen C, Madsbad S. Insulin resistance, the metabolic syndrome, and risk of incident cardiovascular disease: a population-based study. J Am Coll Cardiol. 2007; 49(21):2112-9.

17. Bonora E, Kiechl S, Willeit J, Oberhollenzer F, Egger G, Meigs JB, Bonadonna $\mathrm{RC}$, Muggeo M. Insulin resistance as estimated by homeostasis model assessment predicts incident symptomatic cardiovascular disease in Caucasian subjects from the general population the bruneck study. Diabetes Care. 2007;30(2):318-24

18. Guerrero-Romero F, Simental-Mendía LE, González-Ortiz M, MartínezAbundis E, Ramos-Zavala MG, Hernández-González SO, Jacques-Camarena $\mathrm{O}$, Rodríguez-Morán M. The product of triglycerides and glucose, a simple measure of insulin sensitivity. Comparison with the euglycemichyperinsulinemic clamp. J Clin Endocrinol Metab. 2010;95(7):3347-51.

19. Abbasi F, Reaven GM. Comparison of two methods using plasma triglyceride concentration as a surrogate estimate of insulin action in nondiabetic subjects: triglycerides $\times$ glucose versus triglyceride/high-density lipoprotein cholesterol. Metabolism. 2011;60(12):1673-6.

20. Vasques ACJ, Novaes FS, de Oliveira MS, Souza JRM, Yamanaka A, Pareja JC, Tambascia MA, Saad MJA, Geloneze B. TyG index performs better than HOMA in a Brazilian population: a hyperglycemic clamp validated study. Diabetes Res Clin Pract. 2011;93(3):e98-100.

21. Lee $\mathrm{S}$, Han K, Yang H, Kim H, Cho J, Kwon H, Park Y, Cha B, Yoon K. A novel criterion for identifying metabolically obese but normal weight individuals using the product of triglycerides and glucose. Nutr Diab. 2015;5(4):e149.

22. Irace C, Carallo C, Scavelli F, Franceschi M, Esposito T, Tripolino C, Gnasso A. Markers of insulin resistance and carotid atherosclerosis. A comparison of the homeostasis model assessment and triglyceride glucose index. Int J Clin Pract. 2013;67(7):665-72

23. Lewis GF, Carpentier A, Adeli K, Giacca A. Disordered fat storage and mobilization in the pathogenesis of insulin resistance and type 2 diabetes. Endocr Rev. 2002;23(2):201-29.

24. Parhofer KG. Interaction between glucose and lipid metabolism: more than diabetic dyslipidemia. Diab Metab J. 2015;39(5):353-62.

25. Kelley DE, Goodpaster BH. Skeletal muscle triglyceride an aspect of regional adiposity and insulin resistance. Diabetes Care. 2001;24(5):933-41.

26. Nagle CA, Klett EL, Coleman RA. Hepatic triacylglycerol accumulation and insulin resistance. J Lipid Res. 2009:50(Supplement):S74-9.

27. Kraegen E, Cooney G, Ye J, Thompson A. Triglycerides, fatty acids and insulin resistance-hyperinsulinemia. Exp Clin Endocrinol Diabetes. 2000; 109(4):S516-26.

28. Scicchitano P, Cameli M, Maiello M, Modesti PA, Muiesan ML, Novo S, Palmiero P, Saba PS, Pedrinelli R, Ciccone MM. Nutraceuticals and dyslipidaemia: beyond the common therapeutics. J Funct Foods. 2014;6:11-32.

29. Onat A, Sarı I, Yazı ı M, Can G, Hergenç G, Avcı Gş. Plasma triglycerides, an independent predictor of cardiovascular disease in men: a prospective study based on a population with prevalent metabolic syndrome. Int J Cardiol. 2006;108(1):89-95.

\section{Submit your next manuscript to BioMed Central and we will help you at every step:}

- We accept pre-submission inquiries

- Our selector tool helps you to find the most relevant journal

- We provide round the clock customer support

- Convenient online submission

- Thorough peer review

- Inclusion in PubMed and all major indexing services

- Maximum visibility for your research

Submit your manuscript at www.biomedcentral.com/submit

) Biomed Central 